\title{
Towards A Smartphone-Aided Electronic ELISA For Real-Time Electrochemical Monitoring
}

\author{
Nikolaos G. Pechlivanidis, Konstantinos I. Papadimitriou, Daniel Evans, \\ Nikolaos Vasilakis and Themistoklis Prodromakis \\ Nanoelectronics \& Nanotechnology Research Group, School of Electronics and Computer Science \\ University of Southampton, Southampton, SO17 1BJ, UK, Email: np3e14@ecs.soton.ac.uk
}

\begin{abstract}
This paper details the design and fabrication of a portable, smartphone-integrated electronic platform, tailored to read-out electronic ELISA (eELISA) data from printed circuit board (PCB)-based sensors. The instrument features eight independent, re-configurable current input channels, each consisting of a low-noise transimpedance amplifier (TIA) and filtering stage coupled to low-noise switch ICs for automatic current range detection. A bipolar 16-bit resolution voltage-input analog-todigital converter (ADC) has been employed for digitisation of converted current values received from the analogue front-end. In addition, a bipolar, 12-bit resolution digital-to-analog converter (DAC) combined with standard three-electrode potentiostats provides wide range biasing voltages to the amperometric sensors. The resulting digital data is transmitted via serial interface to an Android-based smartphone, where an ergonomic user interface guides the operator through the detection process. The customised Android application (App) provides real-time monitoring of the electrochemical cell and stores returned biochemical data on the device once measurement is complete.
\end{abstract}

Keywords-Amperometry, Analog-to-Digital Conversion, Bioinstrumentation, eELISA, Lab-on-PCB, Smartphone-Aided

\section{INTRODUCTION}

The modern healthcare system relies heavily upon advanced diagnostic devices. These devices have developed rapidly during the last decade with costs reducing significantly [1]. In addition considerable progress has been made in characterisation of prognostic and diagnostic biomarkers, allowing detection and monitoring of biological samples with improved resolution and in much lower concentrations. In the last decade a significant number of approaches to infectious disease diagnosis have been attempted at point-of-care (PoC), exploiting a large variety of techniques and materials [2], [3]. The lab-on-PCB (LoPCB) paradigm is an attractive method for inexpensive electrochemical biosensing, exploiting exclusively commercial PCB manufacturing processes for low-cost fabrication of biosensors and integrated microfluidics [4]-[6].

Electrochemical detection can be readily employed in diagnostic methodologies through the translation of biological reporter signals to electrical signals. The latter signals can then be digitised and processed appropriately. Furthermore, with the inclusion of microfluidic sample processing, electrochemical biosensors require smaller sample volumes and demonstrate improved assay speed through reduction of required diffusion times. Enzyme-Linked Immuno-Sorbent Assay (ELISA) with colorimetric detection remains the prevailing technique in medical diagnostics, with the majority of contemporary advancement focusing on high-throughput sample processing and automation [7]. However, reporter components of enzymatic assays often provide signals detectable by electrochemical means, eliminating bulky spectrophotometric apparatus. Herein, we employ microfluidic amperometric detection of $\mathrm{H}_{2} \mathrm{O}_{2}$ using LoPCB sensors manufactured entirely by standard commercial techniques to demonstrate a unique PoC approach to medical diagnostics.

Our previous work, introduced the "eELISA" concept, and has validated the efficacy of our sensors by returning data comparable to colorimetric systems with dramatically reduced equipment size, costs and improved assay timings [8]. Herein, we present a novel iteration of our sensor including microfluidic sample control with acquired data reported via a novel eight-channel smartphone-linked bioinstrumentation board, capable of detection across four distinct amperometric ranges. We conceive a versatile and miniaturised diagnostic architecture for integration into standard smartphone devices, providing universal low-cost molecular detection technology.

Section II discusses the proposed smartphone-aided " $e E L$ $I S A$ " system by presenting the PCB-based sensor and the fabricated bioinstrumentation board. In Section III experimental results are shown from the initial characterisation of the board with a high-precision Keithley 6221 current source and two electrochemical assays. Finally, in Section IV we offer some conclusions and future prospects for the presented technology.

\section{The SMARTPhone-Aided "eELISA" System}

This work demonstrates the capability of a portable bioinstrumentation board that receives amperometric signals from our PCB-based sensors. The signal is then digitised and relayed to a smartphone by standard serial protocol. The board is powered by the smartphone and a bespoke App processes received data to display signals in real-time over a pleasing graphical interface. Data can be stored in internal smartphone memory allowing the user to send or share data with other users or clinical staff. As such, this system lends itself favourably to the generation of sub-clinical prognostic metadata that could be evinced through App communication with a central database. The board covers a wide range of the input signal (from -2.5 $\mathrm{mA}$ to $+2.5 \mathrm{~mA}$ ) with adequate resolution, it is lightweight and portable, and the App can run on any modern Android smartphone (version 5.0 or later).

\section{A. Design \& Fabrication of the Bioinstrumentation Board}

The fabricated bioinstrumentation board needs to have certain properties, in order to fully comply with the requirements 
of the "eELISA" technique and smartphone integration. It needs to have relatively small footprint, minute power demands and be able to read electrochemical data accurately and in a low-noise manner [9]. Taking the above into consideration, a careful selection of off-the-shelf components has been made. The analogue front-end of the board features eight low-noise amplifiers (LMP7704) configured in a standard TIA topology, coupled to low-leakage, small $R_{O N}$ resistance switch ICs, able to provide four distinct amperometric sensing ranges. A fully-differential, eight channel, 16-bit resolution voltage-input ADC has been employed for the digitisation of the converted current values received from the analogue front-end. The ADC operates with a bipolar power supply $( \pm 2.5 \mathrm{~V})$ allowing the detection of both reduction and oxidation currents of electrochemical reactions. The digitised data are subsequently been sent to a USB-to-UART interface chip (FT232R) and then to the coupled smartphone device. Finally, for the appropriate biasing of the PCB-based amperometric sensors, a 12-bit DAC (AD5724) has been employed, connected to standard threeelectrode potentiostat circuitry. A simplified schematic diagram of the proposed circuit architecture can be found in Figure 1. The fabricated 4-layer PCB is shown in Figure 2.

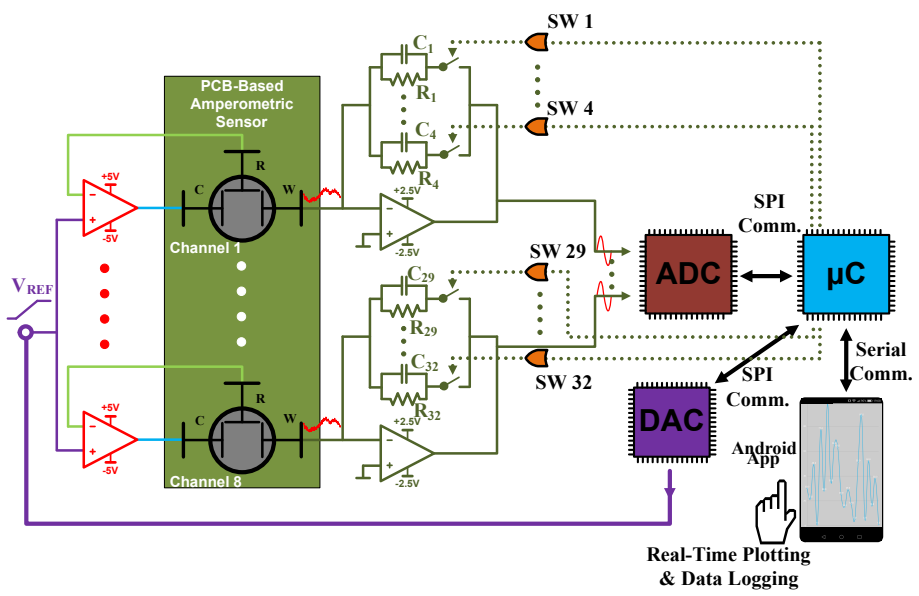

Fig. 1. A simplified schematic diagram of the fabricated bioinstrumentation board that interfaces with the PCB-based sensor.

Using a $32 \mathrm{MHz}$ ATxmega microcontroller, the system is able to constantly monitor the current ranges of the electrochemical reaction and "select" the optimum detection range by activating appropriate switch ICs, which employ optimal value feedback resistors to the TIA setup. All 8 current input channels are fully independent, therefore, they can read multiple reaction currents simultaneously from different sensors operating at different ranges. In addition, the board offers the option to the user to perform a cyclic voltammetry (CV) calibration before every measurement, if necessary, by sweeping the voltage of the on-board DAC from positive $(+2.5 \mathrm{~V})$ to negative values $(-2.5 \mathrm{~V})$ and vice versa. A summary of the board characteristics can be found in the inclusive Table I.

\section{B. PCB-Based Sensing Platform}

The sensing platform consists of working, counter and reference electrodes embedded at the PCB surface during standard manufacturing processes. Working and counter electrodes are gold while the silver reference electrode is converted to

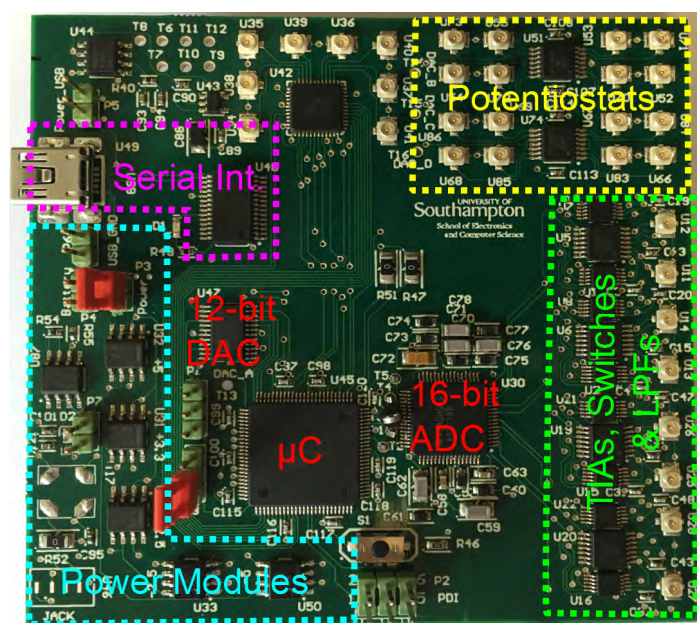

Fig. 2. The smartphone-aided "eELISA" bioinstrumentation board.

silver chloride by treatment with $30 \%$ Sodium Hypochlorite, ensuring its stability throughout the presented experiments. For this analysis a PMMA mould is used to cover the sensing area while PDMS is poured onto the PCB to produce fluidic wells. A PMMA cover encloses the system to generate an amperometric sensing cell. The whole assembly is clamped within an aluminium frame. Sample is introduced through a port in the PMMA cover and removed through an integrated via in contact with the working and reference electrodes. Three alternative working electrode positions are included to improve performance across a wide range reporter component concentrations. The sensing platform is shown in Figure 3.

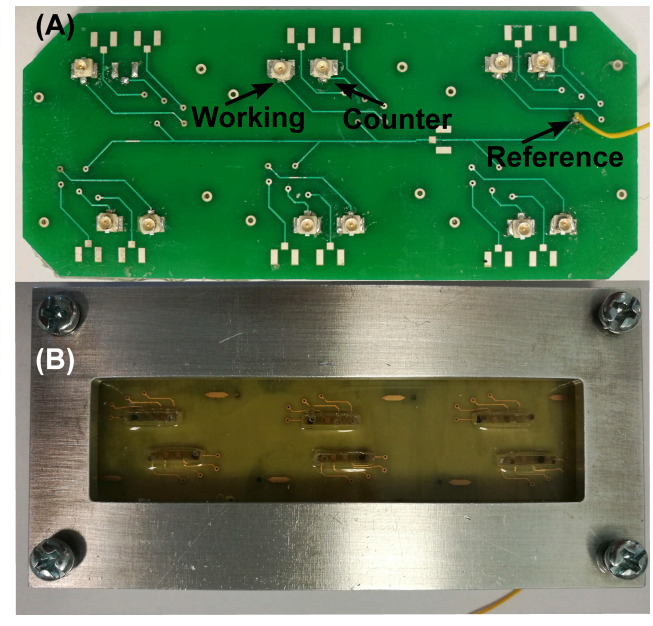

Fig. 3. The proposed PCB-based sensing platform. (A) A top view of the sensor with its three electrodes. (B) The final assembly clamped within an aluminium frame.

Finally, Figure 4 shows two snapshots of the designed App for the smartphone-aided "eELISA". On the left side, the welcoming screen can be seen, where the user can add personal identification details. The right side illustrates a snapshot of a real-time electrochemical measurement. The App allows the storage of biochemical data on the device's internal or external memory, once measurement is complete. 
TABLE I. BIOINSTRUMENTATION BOARD'S CHARACTERISTICS

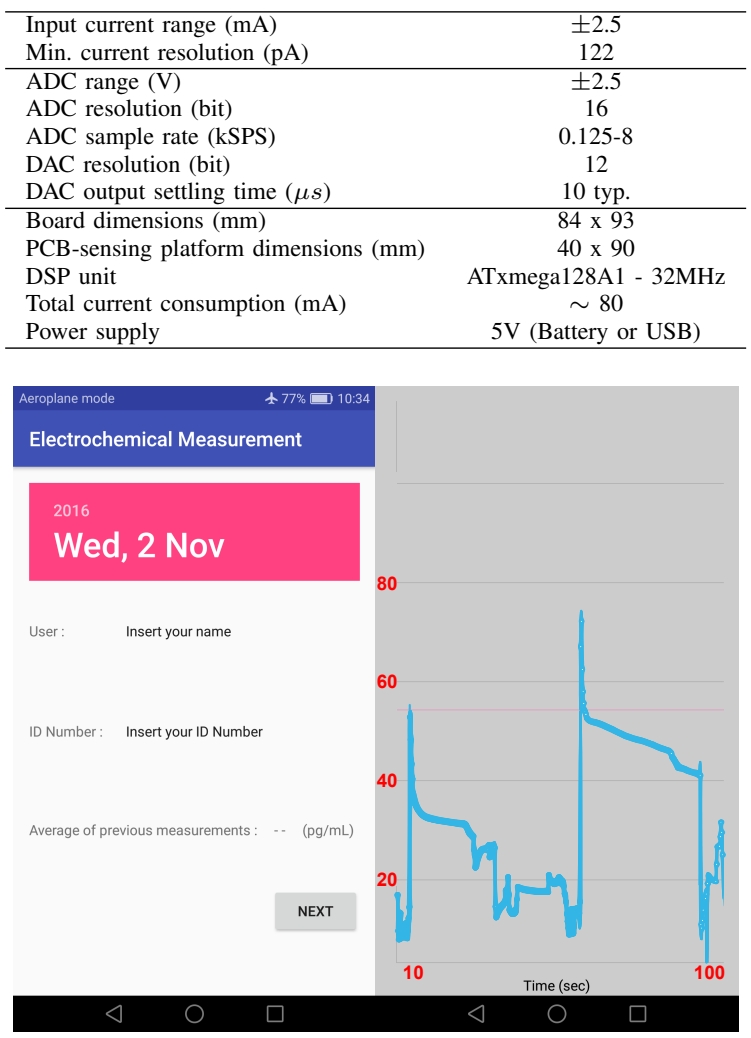

Fig. 4. Two snapshots of the designed Android-based App. On the left, the welcoming screen can be seen, while on the right a snapshot of a real-time $\mathrm{NaCl}$ measurement can be found.

\section{EXPERIMENTAL RESULTS}

\section{A. Board Characterisation}

For initial electronic characterisation of the instrument, a high-precision Keithley 6221 AC/DC current source has been used. Primarily, the limits of each current sensing range have been investigated. Although, Table II provides the theoretical read-out limits of the instrument based on the ADC's capabilities $\left(V_{\operatorname{Ref}} /\left(2^{15}-1\right)\right)$, it was vital to identify the actual limitations of the device. Adequate resolution is an important board's feature, therefore, in Figure 5, low current measurements have been recorded, using the $4^{\text {th }}$ range of the board $\left(R_{4}=1 \mathrm{M} \Omega\right)$. Step changes of $500 \mathrm{pA}$ and $1 \mathrm{nA}$ have been used on a 100nA baseline DC signal. These step changes correspond to a resolution of $0.5 \%$ and $1 \%$ of the base signal, respectively. The recorded signals demonstrate clear distinction between each step. In addition, a current sweep experiment has been performed. The current source has been programmed to generate discrete steps from $1 \mathrm{nA}$ to $50 \mu \mathrm{A}$, in an attempt to demonstrate in real-time the automatic detection range algorithm of the board. The results are shown in Figure 6.

TABLE II. SUMMARY OF THE CHARACTERISATION EXPERIMENTS

\begin{tabular}{lcc}
\hline & \multicolumn{2}{c}{ Programmable Current Ranges } \\
\cline { 2 - 3 }$R_{1}=1 \mathrm{~K} \Omega$ & Current Range & Resolution \\
$R_{2}=10 \mathrm{~K} \Omega$ & $\pm 2.5 \mathrm{~mA}$ & $122 \mathrm{nA}$ \\
$R_{3}=100 \mathrm{~K} \Omega$ & $\pm 250 \mu \mathrm{A}$ & $12.2 \mathrm{nA}$ \\
$R_{4}=1 \mathrm{M} \Omega$ & $\pm 25 \mu \mathrm{A}$ & $1.22 \mathrm{nA}$ \\
\hline
\end{tabular}

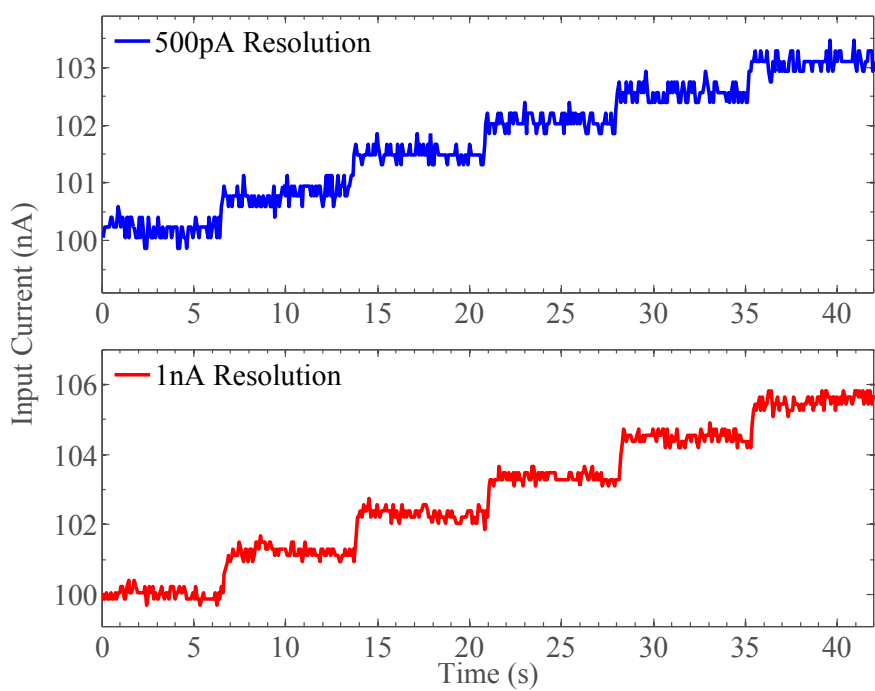

Fig. 5. Investigation of the resolution properties of the instrument. The graphs demonstrate adequate, visible readings with $500 \mathrm{pA}$ and $1 \mathrm{nA}$ step changes.

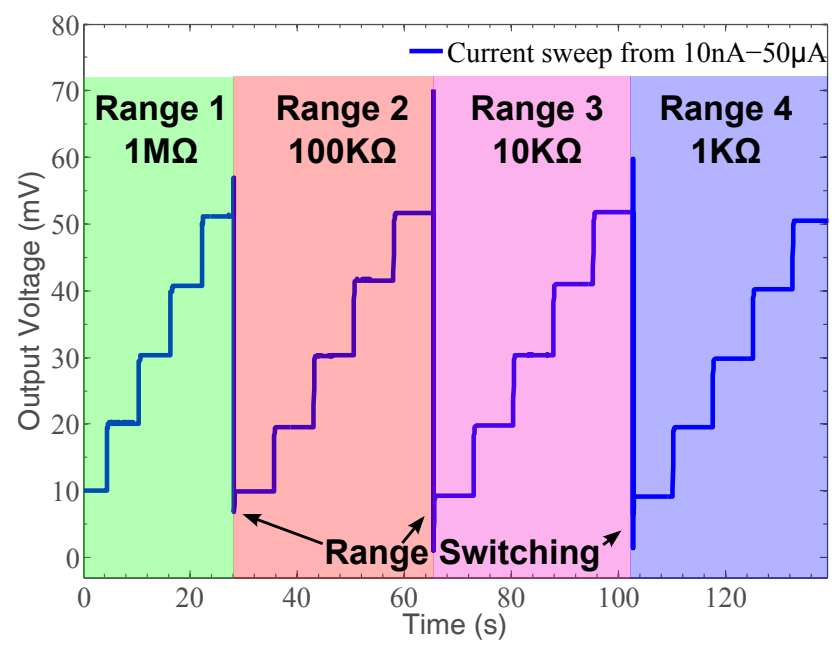

Fig. 6. Automatic adaptation of the instrument's current reading range, as the input current increases from $10 \mathrm{nA}-50 \mu \mathrm{A}$. The glitches at the output are due to range changes of the Keithley 6221 current source driving the input.

\section{B. Assay Data}

$\mathrm{NaCl}$ concentration was assayed amperometrically across a range of concentrations to complement data showing bioinstrumentation processing of artificially generated signals. A $500 \mathrm{mM} \mathrm{NaCl}$ solution was diluted across ten samples in a $\log _{2}$ dilution scheme. Samples were introduced to the sensor of Figure 2 via the upper port and cleared from the system through a trans-PCB via. We observed linear relationships between $\mathrm{NaCl}$ concentration and current magnitude for high and low salt concentration schemes (see Figure 7). However it could be argued that when viewed together the data suggests an exponential relationship, which is hard to explain within a conventional amperometric model but likely results from signal modulations effected by the inclusion of both an oxidative system at the anode and a chloride ion, leading to corrosion at the gold surface proportional to chloride ion concentrations thus effectively amplifying the amperometric signal. 
Many colorimetric detection systems employ a $3,3^{\prime}, 5,5^{\prime}$ Tetramethylbenzidine (TMB) substrate converted to a di-imine product by the horseradish peroxidase (HRP) enzyme. HRP is conjugated to detect antibodies and is thus proportional to target biomarker concentrations in immunochemical assay systems. In the enzymatic conversion of TMB, HRP consumes $\mathrm{H}_{2} \mathrm{O}_{2}$, so in excess substrate $\mathrm{H}_{2} \mathrm{O}_{2}$ concentrations are also proportional to those of the target biomarker. Using the electrochemical cell and fluidics described previously, we demonstrate amperometric signal magnitude proportional to titrated $\mathrm{H}_{2} \mathrm{O}_{2}$ concentrations. $\mathrm{H}_{2} \mathrm{O}_{2}$ was diluted in $10 \mathrm{mM}$ HEPES buffer from a top concentration of $10 \mathrm{mM}$ across four points in a $\log _{2}$ scheme. Amperometric assessment returns a broadly linear relationship between $\mathrm{H}_{2} \mathrm{O}_{2}$ concentration and measured current, as shown in Figure $8 . \mathrm{H}_{2} \mathrm{O}_{2}$ concentrations represent standard colorimetric HRP assay concentrations as defined by commercial TMB-assay-specific Phosphate-Citrate buffer containing $0.014 \%(6 \mathrm{mM})$ Urea Hydrogen Peroxide (Sigma-Aldrich P4560). TMB is a common reporter for ELISA colorimetric detection, thus, this detection system is appropriate for the replacement of colorimetry in standard TMB-based diagnostics.

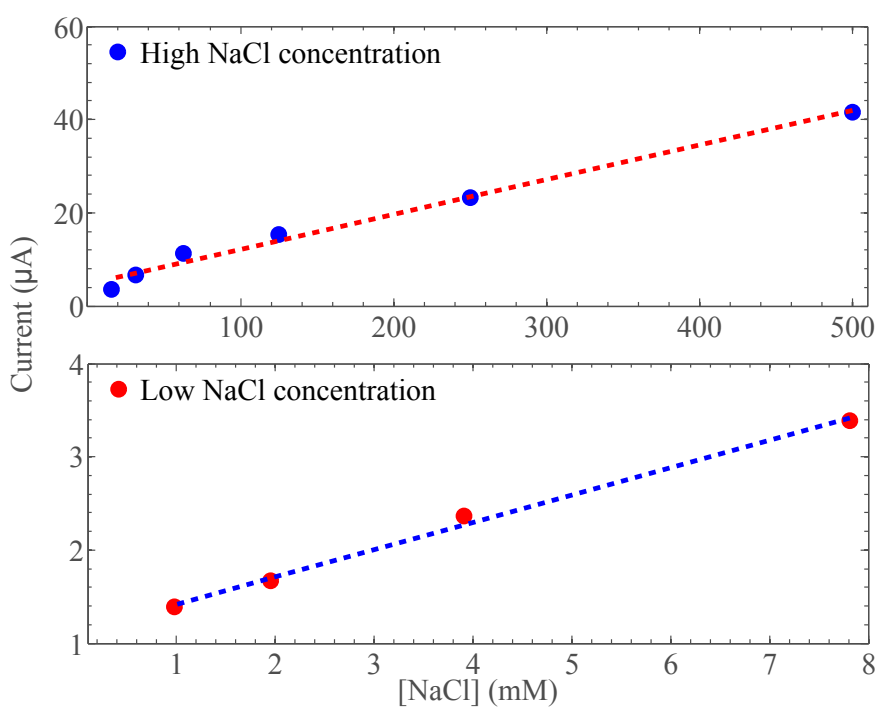

Fig. 7. Amperometric measurement of $\mathrm{NaCl}$ concentrations (high and low) with the fabricated board. The system exhibits high degree of linearity with coefficients of determination $\left(R^{2}\right)$ equal to 0.9883 and 0.9950 for the high and low $\mathrm{NaCl}$ concentration measurements, respectively.

\section{CONCLUSION}

We present a portable diagnostic framework suitable for measurement of $\mathrm{H}_{2} \mathrm{O}_{2}$ at concentrations relevant to industry standard colorimetric assay protocols. We demonstrate smartphone connectivity from the phone's USB port, through a standard serial interface. The device is portable and power overheads are easily satisfied using a standard smartphone battery. We demonstrate amperometric measurement capability in four distinct regions between $-2.5 \mathrm{~mA}$ and $+2.5 \mathrm{~mA}$ with sufficient resolution. The associated App is clear and userfriendly. Successful demonstration of amperometric sensing cells, signal digitisation and processing via the bioinstrumentation platform, smartphone integration and ergonomic read-

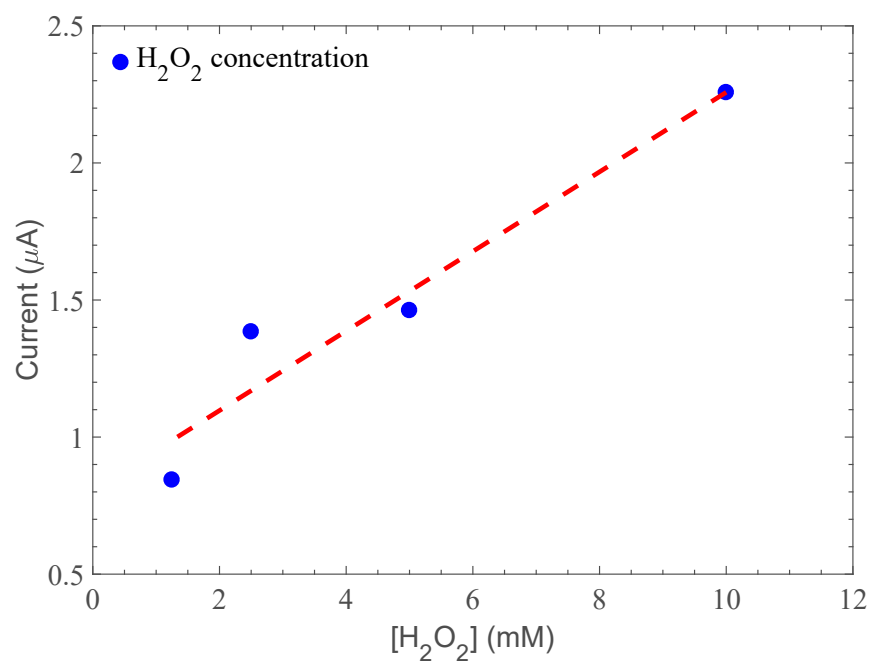

Fig. 8. Amperometric measurement of $\mathrm{H}_{2} \mathrm{O}_{2}$ concentrations, using the PCBbased sensor of Figure 3. Again. the system exhibits high degree of linearity with coefficient of determination $\left(R^{2}\right)$ equal to 0.9240 .

out confirm the feasibility of a future fully integrated PoC diagnostic technology, including PCB-based microfluidics [6].

\section{ACKNOWLEDGMENT}

The authors acknowledge the financial support of the Engineering and Physical Sciences Research Council (EPSRC), EP/L020920/1 research grant.

\section{REFERENCES}

[1] J. Howick, J. W. Cals, C. Jones, C. P. Price, A. Plüddemann, C. Heneghan, M. Y. Berger, F. Buntinx, J. Hickner, W. Pace et al., "Current and future use of point-of-care tests in primary care: an international survey in australia, belgium, the netherlands, the uk and the usa," BMJ open, vol. 4, no. 8, p. e005611, 2014.

[2] E. Ghafar-Zadeh, "Wireless integrated biosensors for point-of-care diagnostic applications," Sensors, vol. 15, no. 2, pp. 3236-3261, 2015.

[3] H. Häkkinen, "The gold-sulfur interface at the nanoscale," Nature chemistry, vol. 4, no. 6, pp. 443-455, 2012.

[4] T. Prodromakis, Y. Liu, J. Yang, D. Hollinghurst, and C. Toumazou, "A novel design approach for developing chemical sensing platforms using inexpensive technologies," in Biomedical Circuits and Systems Conference (BioCAS), 2011 IEEE, Nov 2011, pp. 369-372.

[5] T. Prodromakis, Y. Liu, and C. Toumazou, "A low-cost disposable chemical sensing platform based on discrete components," Electron Device Letters, IEEE, vol. 32, no. 3, pp. 417-419, 2011.

[6] N. Vasilakis, K. I. Papadimitriou, D. Evans, H. Morgan, and T. Prodromakis, "The Lab-on-PCB Framework for Affordable, Electronic-Based Point-of-Care Diagnostics: from Design to Manufacturing," in IEEE-NIH 2016 Special Topics Conference on Healthcare Innovations and Pointof-Care Technologies. IEEE, 2016.

[7] A. Bhimji, A. A. Zaragoza, L. S. Live, and S. O. Kelley, "Electrochemical enzyme-linked immunosorbent assay featuring proximal reagent generation: Detection of human immunodeficiency virus antibodies in clinical samples," Analytical chemistry, vol. 85, no. 14, pp. 6813-6819, 2013.

[8] K. I. Papadimitriou, D. Evans, H. Morgan, and T. Prodromakis, "A PCBbased electronic ELISA system for rapid, portable infectious disease diagnosis," in Biomedical Circuits and Systems Conference (BioCAS), 2016 IEEE. IEEE, 2016, pp. 1-4.

[9] K. I. Papadimitriou, C. Wang, M. L. Rogers, S. A. Gowers, C. L. Leong, M. G. Boutelle, and E. M. Drakakis, "High-performance bioinstrumentation for real-time neuroelectrochemical traumatic brain injury monitoring," Frontiers in Human Neuroscience, vol. 10, p. 212, 2016. 